Review

\title{
Effect of Cardio-Metabolic Risk Factors Clustering with or without Arterial Hypertension on Arterial Stiffness: A Narrative Review
}

\author{
Vasilios G. Athyros *, Andromachi Reklou, Antonis Lazarides, Eudoxia Mitsiou and \\ Asterios Karagiannis \\ Department of Internal Medicine, Medical School, Aristotle University of Thessaloniki, Hippokration \\ Hospital, 15 Marmara St, Thessaloniki, 55132, Greece; E-Mails: machirk128@yahoo.com (A.R.); \\ spanbiol@hotmail.com (A.L.); mitsevi@gmail.com (E.M.); askar@med.auth.gr (A.K.) \\ * Author to whom correspondence should be addressed; E-Mail: athyros@med.auth.gr or \\ vathyros@gmail.com; Tel.: +30-2310-892-606; Fax: +30-2310-835-955.
}

Received: 20 June 2013; in revised form: 9 November 2013 / Accepted: 14 November 2013 / Published: 20 November 2013

\begin{abstract}
The clustering of cardio-metabolic risk factors, either when called metabolic syndrome (MetS) or not, substantially increases the risk of cardiovascular disease (CVD) and causes mortality. One of the possible mechanisms for this clustering's adverse effect is an increase in arterial stiffness (AS), and in high central aortic blood pressure (CABP), which are significant and independent CVD risk factors. Arterial hypertension was connected to AS long ago; however, other MetS components (obesity, dyslipidaemia, dysglycaemia) or MetS associated abnormalities not included in MetS diagnostic criteria (renal dysfunction, hyperuricaemia, hypercoaglutability, menopause, non alcoholic fatty liver disease, and obstructive sleep apnea) have been implicated too. We discuss the evidence connecting these cardio-metabolic risk factors, which negatively affect AS and finally increase CVD risk. Furthermore, we discuss the impact of possible lifestyle and pharmacological interventions on all these cardio-metabolic risk factors, in an effort to reduce CVD risk and identify features that should be taken into consideration when treating MetS patients with or without arterial hypertension.
\end{abstract}


Keywords: metabolic syndrome; arterial stiffness; central aortic pressure; arterial hypertension; cardiovascular disease; type 2 diabetes mellitus; chronic kidney disease; hyperuricaemia; non alcoholic fatty liver disease; obstructive sleep apnea

\section{Abbreviation List}

$2-\mathrm{HPG}=2 \mathrm{~h}$ plasma glucose, following a $75 \mathrm{~g}$ oral glucose tolerance test;

ACE-I = angiotensin enzyme inhibition; ARBs = angiotensin II receptor blockers;

AS $=$ arterial stiffness; ASCOT $=$ Anglo-Scandinavian Cardiac Outcomes Trial;

$\mathrm{AT} 1 \mathrm{R}=$ angiotensin II type 1 receptor; $\mathrm{BMI}=$ body mass index;

$\mathrm{BP}=$ blood pressure; $\mathrm{CABP}=$ central aortic blood pressure;

$\mathrm{CAFE}=$ Central Artery Function Evaluation study; $\mathrm{CKD}=$ chronic kidney disease;

GLP-1 agonist = glucagon-like peptide-1 agonist;

$\mathrm{CPAP}=$ continuous positive airway pressure therapy;

$\mathrm{CVD}=$ cardiovascular disease; $\mathrm{DBP}=$ diastolic blood pressure;

e-GFR = estimated glomerular filtration rate; ESRD = end stage renal disease;

$\mathrm{FPG}=$ fasting plasma glucose; HDL-C $=$ high density lipoprotein cholesterol;

HOMA = homeostatic model assessment; IFG = impaired fasting glycaemia

$\mathrm{IR}=$ insulin resistance; LDL-C $=$ low-density lipoprotein cholesterol;

$\mathrm{LV}=$ left ventricle; MetS = metabolic syndrome; NAFLD = non-alcoholic fatty liver disease;

$\mathrm{NO}=$ nitric oxide; OSA = obstructive sleep apnea; PCOS = polycystic ovary syndrome;

$\mathrm{PP}=$ pulse pressure; $\mathrm{PWV}=$ pulse wave velocity; RAAS = renin-angiotensin-aldosterone system;

$\mathrm{SBP}=$ systolic blood pressure; $\mathrm{SUA}=$ serum uric acid;

$\mathrm{T} 2 \mathrm{DM}=$ type 2 diabetes mellitus; $\mathrm{WC}=$ waist circumference

\section{Introduction}

Arterial compliance plays a key role in the function of the cardiovascular system, given the intermittent contraction of left ventricle (LV) and the endless need of organs and tissues for oxygen and nutrients [1]. The contraction of the LV increases the pressure in large vessels (i.e., the aorta), which, because of their elasticity, store a significant part of the blood ejected from LV contraction. After the closure of the aortic valve, the recoil of large vessels during LV diastole pushes the blood towards the periphery, keeping a steady flow to organs and tissues [1].

Arterial stiffness (AS) and its hemodynamic consequences have been established as predictors of adverse cardiovascular disease (CVD) outcomes [1,2]. AS is closely related to systolic hypertension and has a causal effect on coronary artery disease, stroke, and heart failure, which are the leading causes of morbidity and mortality in western countries [2-4]. Although blood pressure (BP) is a major determinant of AS, the latter has an additional predicative potential over and above that of brachial BP, and may be affected by CVD risk factors other than arterial hypertension [5]. 


\section{Methodology}

The research question was: Are all disease conditions related to what is usually described as metabolic syndrome (MetS) considered to be components of the syndrome or are they just related with it in most cases, and have an adverse effect on AS despite the presence of arterial hypertension, which along with age are the key factors for stiff arteries?

Many researchers use several definitions of MetS, they use several methods to measure AS, have different endpoints, and there are no studies at all that investigate the relation of all or even the majority of disease states not included in the MetS criteria with AS. As such, it was not possible to write a systemic review on this issue, and we chose to write a narrative review on the subject at hand. This was done because the issue of AS is an important independent CVD risk factor and the attending physician should deal with it if the higher possible reduction of CVD risk is the aim of any intervention in MetS.

Narrative reviews are most useful for obtaining a broad perspective on a topic, including sections on the physiology and/or epidemiology of a topic. Authors search for pivotal papers known to the subject expert and respond with their results to review the hypothesis or question. Systemic review and meta-analysis and are not in direct antagonism with narrative reviews. Where systemic review metaanalysis is applicable (many studies available that test the same hypothesis), it is generally the preferred method. However, in this case there are not enough studies with the same hypothesis or methods or endpoints.

The literature search strategy was: Studies evaluating relationships of "MetS" or "arterial hypertension" or "glucose intolerance" or "menopause" or "chronic kidney disease" or "hyperuricaemia" or "hypercoagulability" or "non-alcoholic fatty liver disease" or "dyslipidaemia" or "small dense LDL particles" or "obstructive sleep apnea" or "treatment" with "stiffness," or "arterial stiffness" or "arterial elasticity," or "pulse wave velocity" or "prediction," or "cardiovascular risk" or "death" or "mortality" or "outcome" or "events" "diabetes mellitus" were drawn from PubMed and Cochrane databases until May 2013. Data sources were also identified through manually searching the references of articles using PubMed.

\section{Treating AS in Patients with a Cluster of MetS Defining and Non Diagnostic Criteria of MetS}

The effective means to treat the cluster of all 12 cardio-metabolic risk factors analyzed below and their combinations, with or without the presence of arterial hypertension show promise for the control of AS, leading to CVD risk reduction.

\subsection{Pathophysiology of $A S$}

The elastic properties of arteries differ along the arterial tree, with more elastic proximal arteries and stiffer distal arteries [6]. AS is largely determined by the structure of the tunica media, which contains the elastic components of the aortic wall, such as elastin fibers and collagen [7]. Several factors influence arterial wall structure. Ageing is closely associated with AS through the fragmentation of elastin fibers, the decrease in elastin/collagen ratio, and the calcification of the tunica media [7]. This process is called arteriosclerosis and should be distinguished from atherosclerosis, 
which is a disease of the intima. Arterial hypertension is a major determinant of arteriosclerosis and arterial stiffening, through changes in the tunica media that take place earlier than that induced by ageing; this is considered to be the "early vascular ageing". However, AS contributes to the worsening of arterial hypertension within a vicious cycle [7]. AS is caused by other CVD risk factors besides arterial hypertension, such as smoking, diabetes mellitus, mainly type 2 (T2DM), hypercholesterolaemia, and chronic kidney disease (CKD) [5,8,9]. Arterial stiffening is also increased in patients with established atherosclerotic CVD [7]. AS is characterized by increased pulse wave velocity (PWV) and early return in the ascending aorta of reflected waves, during the systolic phase of the pressure waveform. Thus, systolic central aortic blood pressure (CABP), and consequently LV afterload, is increased. These induce LV hypertrophy (and stiffness) and predispose to LV systolic and diastolic dysfunction [7]. Furthermore, in patients with AS central CABP during the diastole is decreased, thus substantially reducing diastolic coronary artery perfusion. The combination of LV hypertrophy and reduced coronary perfusion leads to myocardial oxygen supply/demand mismatch, and further deteriorates LV systolic and diastolic function [10]. This combination of high systolic and low diastolic CABP leads to an increased value of pulse pressure (PP), which expresses AS and appears to be the most powerful measure to identify those hypertensive patients at greatest CVD risk [10].

Among the different non-invasive methods used to assess arterial stiffness is the carotid-femoral PWV has emerged as a gold standard due to its accuracy, reproducibility, relative easy measurement, and low costs. Furthermore, PWV has yielded prognostic value beyond and above traditional risk factors. PWV is assessed non-invasive using the Complior, SphygmoCor, Arteriograph or the Vicorder systems with respective software. Electrocardiogram-gated carotid and femoral waveforms were recorded using applanation tonometry. Carotid-femoral path length was measured as the difference between the surface distances joining the suprasternal notch, the umbilicus and the femoral pulse and the suprasternal notch and the carotid pulse [11]. Carotid-femoral transit time was estimated in 8-10 sequential femoral and carotid waveforms as the average time difference between the onset of the femoral and carotid waveforms. The foot of the pulse wave was identified using the intersecting tangent method. PWV was calculated as the carotid-femoral path length divided by the carotid-femoral transit time. This is an established, non-invasive and reproducible method to determine arterial stiff-ness and no adjustments are required for transit time and path length. Most studies use data on PWV deriving from 16,867 subjects and patients from 13 different centers across eight European countries [11]. Of these, 11,092 individuals were free from overt CV disease, did not have diabetes and were untreated by either anti-hypertensive or lipid-lowering drugs and constituted the reference value population. Prior to data pooling, PWV values were converted to a common standard using established conversion formulae. Subjects were categorized by age and further subdivided according to BP categories. PWV increased with age and BP category; the increase with age being more pronounced for higher BP categories and the increase with BP being more important for older subjects. The distribution of PWV with age and BP category is described and reference values for PWV are established. Normal values are proposed based on the PWV values observed in the non-hypertensive subpopulation with no additional CVD risk factors. Normal median values according to age were 6.0 (range 5.2-7.0) $\mathrm{m} / \mathrm{s}$ in $<30$ years old and $10.1(7.6-13.8) \mathrm{m} / \mathrm{s}$ for subjects $>75$ years old. If participants had $\mathrm{AH}$, median values ranged from $7.2 \mathrm{~m} / \mathrm{s}$ to $13.5 \mathrm{~m} / \mathrm{s}$ respectively [11]. Among an adult population that varies widely in age, brachial ankle (baPWV) is significantly and independently associated with aortic PWV 
as well as leg PWV. Second, decreases in aortic PWV with exercise intervention are related to the corresponding changes in baPWV [12]. These results suggest that baPWV may provide qualitatively similar information to those derived from aortic PWV, a well-established index of central arterial stiffness. In male subjects, the upper limits of baPWV values were 1497/1425, 1518/1513, 1716/1726, $1925 / 1971$, and $2310 / 2115 \mathrm{~cm} / \mathrm{s}$, obtained using two different statistical methods for the age groups of 30-39, 40-49, 50-59, 60-69, and 70 and older, respectively [12]. For females, the upper limits of baPWV values were 1427/1411, 1559/1499, 1733/1739, 1958/1974, and 2721/2577 cm/s for the age groups of 30-39, 40-49, 50-59, 60-69, and 70 and older, respectively [12].

\subsection{Metabolic Syndrome (MetS)}

The MetS is a cluster of CVD and T2DM risk factors [11]. Patients with MetS are at higher risk for vascular events [12,13] and all-cause mortality [14], even in the absence of clinically evident CVD and/or T2DM [15,16].

However, there are concerns suggesting that when confounding factors such as obesity are accounted for, diagnosis of the MetS has a negligible association with the risk of CVD [17]. This approach encouraged the American Diabetes Association and the European Association for the Study of Diabetes to issue a joint statement identifying concerns on the clinical utility of MetS as a syndrome [18].

At the end of the day, all parts recognise that the clustering of several CVD risk factors, regardless if they are considered as a syndrome or not, confer an increased CVD risk, even if that is the just the sum of the risks of MetS components. In 2006 the International Diabetes Federation [19] released a worldwide definition of MetS with the following criteria: central obesity [defined as waist circumference (WC) with ethnicity-specific values] plus any two of the following: raised triglycerides: $>150 \mathrm{mg} / \mathrm{dL}$, or specific treatment for this lipid abnormality, reduced high density lipoprotein cholesterol (HDL-C): $<40 \mathrm{mg} / \mathrm{dL}$ in males, $<50 \mathrm{mg} / \mathrm{dL}$ in females, or specific treatment for this lipid abnormality, raised $\mathrm{BP}$ : systolic $\mathrm{BP}>130$ or diastolic $\mathrm{BP}>85 \mathrm{~mm} \mathrm{Hg}$, or treatment of previously diagnosed arterial hypertension, raised fasting plasma glucose (FPG): $>100 \mathrm{mg} / \mathrm{dL}$, or previously diagnosed T2DM [19].. In this consensus, obesity is characterized as the main clinical manifestation of MetS and insulin resistance (IR) as the central pathophysiological aberration. There are another 5 MetS definitions. However it is not the aim of this review to investigate and compare the differences of various MetS definitions, because the review is not about MetS and AS, but goes beyond that analyzing in total the effect of 12 cardio-metabolic risk factors and their combinations.

\subsection{AS in MetS with Arterial Hypertension}

It has been proposed that IR induces AS, which in tern leads to increased CVD risk [20]. Prospective studies have shown that patients with the IR syndrome have a greater increase in AS than age-matched controls without the syndrome [21]. Depletion of nitric oxide (NO) or ineffectiveness of NO mediated vasodilatation associated with the progression of IR to T2DM may induce increased AS [22]. Also increased release of endothelin-1 leads to impaired structural properties of the vessel wall [22]. As a result, the renin-angiotensin-aldosterone system (RAAS) is activated in the diseased vascular beds, with up regulation of the angiotensin II type 1 receptor (AT1R). This results in arterial hypertension, which is a component of MetS, while the increased AT1R mediated activity in the 
vasculature is central to the development of increased AS, which is further deteriorates IR within a vicious cycle [22]. Intervention that aims at AT1R blockade [such as angiotensin receptor inhibitors (ARBs)] may, therefore, be a valuable treatment for early IR, as antagonism of AT1 receptors is beneficial by itself and also allows angiotensin II to act unopposed on AT2R, activation of which is beneficial in treating arterial hypertension and preventing early AS [22]. Moreover, there is data suggesting that in hypertensive, non-diabetic, older adults, IR is associated with AS, independent of glucose tolerance status [23]. In addition, IR is associated with AS, independent of age, baseline systolic BP (SBP), gender and metabolic status [24]. All the above suggest that MetS through IR, its main pathophysiological aberration, induces AS, regardless of age, gender, arterial hypertension or glycaemic control.

\subsection{AS in MetS with Glucose Intolerance or T2DM}

Dysglycaemia is one of MetS components in all definitions. A meta regression analysis of data from 20 studies with 95,783 individuals followed for 12.4 years showed that there is a continuous positive relationship between FPG and $2 \mathrm{~h}$ plasma glucose, following a $75 \mathrm{~g}$ oral glucose tolerance test (2-HPG), with incident CVD events [25]. The 2-HPG is a better predictor of death from all causes and CVD than FPG [26]. There also data on an independent association between the impaired fasting glycaemia (IFG), impaired glucose tolerance (IGT), and T2DM with early AS as well as with total and CVD morbidity and mortality [27,28]. It seems that structural mechanisms connect hyperglycaemia with vascular complications even within prediabetes thresholds [27,28]. These relationships are reported as a cumulative effect of MetS on AS as an entity [29-31], while there are relative independent strengths of FPG and 2-HPG associations with AS [32,33]. Thus, it seems that there is a clear relationship of premature arterial stiffening with glucose metabolism disorders, within or outside the T2DM range [33], in MetS patients with or without arterial hypertension.

\subsection{AS in MetS and Menopause}

Postmenopausal women tend to have MetS because of hormonal and metabolic changes [34]. CVD incidence increases after menopause and this may be related, at least in part, to IR and AS [34]. From the 9,555 postmenopausal (women) participants of a study, 455 had MetS and normal glucose and BP levels [34]. In addition to a full biochemical screening, adiponectin and PWV (to assess AS) were evaluated. IR was estimated by the homeostatic model assessment (HOMA)-IR. The women were stratified into 3 groups according to their HOMA-IR values. There were significant differences in metabolic parameters between the 3 groups. The mean age, body mass index (BMI), WC, FPG, TG, SBP, diastolic BP (DBP), aortic PWV, and peripheral PWV were all increased according to the degree of IR [34]. Moreover, HDL-C and adiponectin levels were decreased according to the degree of IR. Age, BMI, FPG, TG, insulin, SBP, HOMA-IR, aortic PWV, and peripheral PWV were significantly higher in women with central obesity, while HDL-C and adiponectin were significantly lower in these women [34]. PWV (aortic and peripheral) were significantly correlated with age, WC, total cholesterol, SBP, DBP, insulin, and HOMA-IR. The results of multiple regression analysis indicated that SBP, DBP, and HOMA-IR were independently correlated with PWV and therefore to AS [34]. In the normoglycaemic and normotensive postmenopausal group IR was independently and significantly 
associated with AS as demonstrated by increased aortic and peripheral PWV [34]. These associations between MetS and AS were also shown in premenopausal women with a cluster of cardio-metabolic risk factors [35].

\section{6. $A S$ in $M e t S$ and $C K D$}

People with the MetS have an increased risk, up to 5 times, of developing CKD in comparison with those without the syndrome [36,37], even in children [38]. A meta-analysis of eleven studies $(n=30,146)$ showed that MetS and its components are associated with the development of estimated glomerular filtration rate (e-GFR) $<60 \mathrm{~mL} / \mathrm{min}$ per $1.73 \mathrm{~m} 2$ (CKD stage 3 or higher) and microalbuminuria or overt proteinuria [36]. Patients with CKD are at an increased risk not only for ESRD but also for CVD [38]. CKD is a CVD predictor and is practically considered as an ischaemic heart disease equivalent, similar to DM [39]. This is due to the independent harmful effect of CKD itself as well as to CKD related CVD risk factors, such as arterial hypertension and dyslipidaemia [40-44]. A major CKD risk factor is increased AS of large arteries, which is independent of other major confounders [39]. Furthermore, increased AS in central arteries may cause further reduction in GFR in subjects with CKD, within a vicious cycle [45]. This suggests that CKD may interact not only with small but also with large arteries, independently of age, BP level, and classical CVD risk factors $[45,46]$. On the other hand, arterial stiffening is an independent predictor of survival in ESRD in the general population [47]. Given that statins play a role in arterial "destiffening" [40-44,48,49], it has been shown that the dose and the compound play a major role in achieving improvement of GFR, while reducing AS and CVD risk [50].

\subsection{AS in MetS and Hyperuricaemia}

Elevated serum uric acid (SUA) levels are related to the presence of MetS, while its levels increase with the increasing number of MetS components (arterial hypertension, dyslipidaemia, DM and visceral obesity) [51], to the degree that many researchers suggest that SUA should be considered a MetS component in a future definition [52].

SUA is also closely related to CKD and is an independent CVD risk factor of total and CVD morbidity and mortality, at least in high risk patients [51-53]. SUA seems to be significantly correlated with central systolic BP and AS in both genders [54]. Therefore, SUA may contribute to the pathogenesis of arterial hypertension, mainly through increased wave reflections and contribute thus to the increase of CVD risk [54]. This was confirmed by a study suggesting that in both genders, SUA levels are associated with AS, and this relationship is independent of other conventional risk factors for MetS and atherosclerotic CVD [55]. Similar were the results of a large study with postmenopausal women [56]. On the contrary, a study that included relatively young men and women suggested that SUA levels are associated with an increase in AS, which differs between men and women. Women might be more susceptible to vascular damage associated with high SUA levels [57]. Subjects with hyperuricaemia had significantly higher carotid-ankle PWV in both genders; however the carotid-femoral PWV was higher only in women [57]. Nevertheless, in a recent study it was reported that among 779 subjects (393 men and 234 women), witch had normal SUA levels, those with high-normal SUA levels had an increased AS (higher brachial-ankle PWV), after adjusting for confounding factors [58]. Thus, SUA was identified as an AS risk factor even with high-normal levels, inside the normal range [58]. 


\subsection{AS in MetS and Hypercoagulability}

It has been shown that the MetS presents relations and inter-relations between metabolic aberrations (in glucose, salt, insulin, and lipid metabolism as well as coagulation factors) and haemodynamic abnormalities (due to RAAS stimulation, sympathetic overactivity, and decreased NO bioavailability), which are followed by anaemia, hypercoagulability, organ ischaemia, AS, arterial hypertension, as well as renal and LV dysfunction [59]. These might be considered to form the "circulatory syndrome" [59,60]. Within or outside this circulatory syndrome frame MetS is related to increased levels of clotting factors (tissue factor, factor VII and fibrinogen) as well as inhibition of the endogenous fibrinolysis (increased plasminogen activator inhibitor-1 and decreased tissue plasminogen activator activity) [60,61]. Fibrinogen levels correlate with lipid [62] and inflammatory parameters [60,62], suggesting an involvement of adipose tissue-generated inflammatory cytokines [60]. Elevated von Willebrand's factor and factor VIII levels combine with endothelial injury, whereas vitamin K-dependent coagulation correlate with triglyceride levels [60]. All the above seem to inter-relate with low grade inflammation and with AS [59,60].

\subsection{AS in MetS and Non-Alcoholic Fatty Liver Disease (NAFLD)}

NAFLD is currently the most common form of chronic liver disease. It affects $20 \%-35 \%$ of general population in western countries, while its prevalence increases up to $70 \%-90 \%$ in people with obesity, MetS or T2DM [63]. NAFLD is considered the hepatic manifestation of MetS; it is closely related to other clinical features of MetS, and thus to CVD risk [64]. Several data have documented that NAFLD associates with other CVD risk factors [64,65]. AS was independently associated with the risk for NAFLD, regardless of classical CVD risk factors [64,65]. Moreover, it was shown in a large study $(\mathrm{n}=4,467)$ that not only the presence but also the degree of NAFLD are associated with AS in non-hypertensive, non-diabetic individuals with or without MetS [66].

\subsection{AS in MetS and Dyslipidaemia}

Dyslipidaemia (low HDL-C and/or high triglycerides) is a MetS component. Patients with hypercholesterolaemia have a higher central pulse pressure and stiffer blood vessels than matched controls, despite similar peripheral BP [67,68]. Low-density lipoprotein cholesterol (LDL-C), not a MetS component, seems to be independently associated with AS, while HDL-C is independently (inversely) associated with AS [69]. Statin therapy leads to a reduction in AS, re-enforcing the concept that stiffness is indeed a modifiable CVD risk factor [67]. It has also been shown that PWV, and therefore AS, is increased in carriers of the lecithin cholesterol acetylo-transferase mutation related to low HDL-C [70]. The triglycerides/HDL ratio, a rough measure of the predominance of small-dense LDL particles over large-buoyant ones [71], is an independent determinant of AS in older adults, younger adults, even in adolescents, particularly in these with central obesity, regardless of the presence of arterial hypertension [72,73]. 


\subsection{AS in MetS and Obstructive Sleep Apnea (OSA)}

OSA is a highly prevalent condition. About $24 \%$ of men aged $30-60$ years and $9 \%$ of adult women are estimated to have OSA; most of them have MetS [74]. The close association between OSA, overweight/obesity and CVD morbidity and mortality is a major health problem [75]. A plethora of studies have indicated a causal relationship between OSA and arterial hypertension, T2DM, and CVD, while the relative risk for all-cause mortality in patients with OSA is 1.5 in comparison to the individuals that do not have OSA [75]. One of the possible mechanisms for this adverse effect of OSA is increased AS [74,76]. Interventional studies showed a substantial improvement in symptoms associated with OSA (daytime sleepiness, snoring, morning headaches, and reduction in BP), in patients receiving continuous positive airway pressure therapy (CPAP) [74,75]. Moreover, the benefits of effective CPAP on reduction of CVD risk and fatal and nonfatal CVD events have been established in multiple large trials [77]. It has also been clearly shown that after CPAP treatment in patients with OSA there was a significant improvement of all indices of AS [74,78-80].

\subsection{AS in MetS: Treatment Options}

After all the distressing data presented above, one question pops up: do we have at our disposal effective means to intervene and tackle the clinical consequences of MetS, and particularly those related to AS? There seem to be several effective tools, from lifestyle advice to pharmacological interventions in AS risk factors, which may substantially contribute to the amelioration of CVD risk, mainly the part related to AS.

Aerobic exercise training is associated with lower AS, but little information is available on the required intensity or duration of physical activity. There are data suggesting that both moderate and vigorous physical activity have favorable effects on AS in postmenopausal women, even after adjustment for expected confounders [81]. In the Amsterdam Growth and Health Longitudinal Study it was shown that lifetime vigorous, but not light-to-moderate, habitual physical activity is favorably associated with increased arterial compliance, even after adjustments for sex-specific coefficients [82]. Moreover, adoption of Mediterranean diet beneficially affects AS, even after adjustments for potential confounders [83]. This effect of Mediterranean diet is probably related to its beneficial impact on CVD risk factors, such as BP, central obesity, and dyslipidaemia [83]. Also, in the Amsterdam Growth and Health Longitudinal Study, mentioned above, it was shown that lower intake of fiber throughout the course of young life (from adolescence to adulthood) is associated with AS in adulthood, after adjustment for sex, height, total energy intake, and other lifestyle variables [84].

Thus, intense physical activity and adoption of a healthy diet reach in fiber (preferably the Mediterranean diet) are powerful tools to prevent or treat MetS and AS, thus reducing CVD risk $[85,86]$. However, non-adherence to long term lifestyle changes is very usual and raises important issues for their effectiveness, particularly in cases that they have to be adopted for a life-time period, given that in all interventions the key determinant of success is the adherence level [87].

The majority of older individuals develop higher CABP mediated by increasing AS. An increased CABP may lead to target organ damage through a variety of mechanisms [88]. Data indicate that lowering brachial arterial BP does not necessarily correlate with equal lowering of CABP or AS. Pulse 
pressure varies throughout the arterial tree, resulting in a gradient between central and peripheral pressure. CVD risk factors affect the pulse pressure ratio, and that central pressure cannot be reliably inferred from peripheral pressure. However, assessment of CAPB may improve the identification and management of patients with elevated CVD risk. However, assessment of CAPB may improve the identification and management of patients with elevated CVD risk. BP lowering drugs can have substantially different effects on central aortic pressures and hemodynamics despite a similar impact on brachial BP [89]. Moreover, CAPB may be a determinant of clinical outcomes, and differences in CAPB may be a potential mechanism to explain the different clinical outcomes between the two BP treatment arms in ASCOT [89]. Radial artery applanation tonometry and pulse wave analysis were used to derive CAPB and hemodynamic indexes on repeated visits [89]. The integral system software was used to calculate an averaged radial artery waveform and to derive a corresponding central aortic pressure waveform using a previously validated generalized transfer function. Aortic pressure waveforms were subjected to further analysis by the SphygmoCor software to identify the time to the peak/shoulder of the first and second pressure wave components during systole. The pressure at the peak/shoulder of the first component was identified as P1 height (outgoing pressure wave), and the pressure difference between this point and the maximal pressure during systole ( $\Delta \mathrm{P}$ or augmentation) was identified as the reflected pressure wave occurring during systole. Augmentation index (AIx), defined as the ratio of augmentation to central pulse pressure, is expressed as a percentage:

$$
\operatorname{Aix}=(\Delta \mathrm{P} / \mathrm{PP}) \times 100
$$

where $\mathrm{P}$ is pressure and $\mathrm{PP}$ is pulse pressure. Pulse pressure amplification (PPA) was expressed as the ratio of CAPB to peripheral (brachial) pulse pressure (PPP): PPA = PPP/CPP [89].

Increased AS, independent of brachial arterial BP, appears to be a novel and independent risk factor for CVD and total mortality [90] in healthy older adults and hypertensive patients [88,91]. These emphasize the need for ambulatory (non invasive) measurement of CABP and AS with equipment recording the 24-h brachial BP, heart rate, and PP too [92]. In a study with 211 patients with uncomplicated arterial hypertension, patients were treated for 1 year [92]. Ambulatory AS index, pulse pressure (PP), and aortic PWV were used to assess the effect of drugs on AS [92]. The results showed that administration of antihypertensive drugs with different hemodynamic profiles, ambulatory AS index and aortic PWV behaved similarly and AS was reduced to the same extent [92]. However, studies in animal models suggest that early brief angiotensin enzyme inhibition (ACE-I) decreased arterial pressure and isobaric wall stiffness in spontaneously hypertensive rats; this was associated with marked alteration of wall structure, while the effects persisted after cessation of early treatment [93]. A meta-analysis of short-term trials in humans showed that ACE-Is were more effective than calcium antagonists and placebo on improving AS [94]. In long-term trials, ACE-I, calcium antagonists, beta-blocker, and diuretic reduced significantly PWV compared to placebo [94]. A review of the effects of antihypertensive drugs suggests that RAAS blockers maintain compelling indications in particular CVD conditions, such as microalbuminuria, LV hypertrophy, and AS. The above provide further support to a drug-based antihypertensive strategy [94]. Moreover, the optimal safety profile of several antihypertensive drugs, in particular of ACE-Is, ARBs and renin inhibitors, allows their use in the very early stages of arterial hypertension, as suggested by recent clinical trials [95]. In a metaanalysis with meta-regression of randomized controlled trials, it was demonstrated that ACE-Is reduce 
AS and wave reflections in patients with different pathological conditions [96]. However, due to the lack of high quality and properly powered randomized controlled trials, it is not clear whether ACE-Is are superior to other antihypertensive agents in their effect on AS [96]. The ability of ACE-Is to reduce AS appears to be independent of their ability to reduce BP [96]. In regard to atenolol, its use as a first choice antihypertensive agent was limited by the results of the Central Artery Function Evaluation (CAFE) study [89]. CAFE was a substudy of the Anglo-Scandinavian Cardiac Outcomes Trial (ASCOT), which examined the impact of two different BP lowering-regimens (the amlodipine+/-perindopril-based $v s$. the atenolol+/-thiazide-based therapy) on CABP and hemodynamics [89]. CAFE showed that despite similar brachial SBP between treatment groups, there were substantial reductions in CABP with the amlodipine regimen [96]. Cox regression analysis showed that CABP and central PP was significantly associated with the composite outcome of total CVD events and development of renal impairment (CKD) in the CAFE study population [96]. These differences in CABP may be a potential mechanism to explain the different clinical outcomes between the 2 BP treatment arms in ASCOT [89]. A post hoc analysis of ASCOT showed that the combination of atorvastatin $(10 \mathrm{mg} / \mathrm{d})$ with the amlodipine+/-perindopril-based therapy produced a huge reduction in CVD events (by 52\%) in comparison to placebo [97], while the combination of atorvastatin $(10 \mathrm{mg} / \mathrm{d})$ with the atenolol+/-thiazide-based therapy produced a much smaller reduction in CVD events (by only 15\%) in comparison to placebo [97]. Given that atorvastatin did not significantly affect the CABP in this large cohort of the CAFE study [97], these results designated the beginning of the end of the use of atenolol as a first choice antihypertensive agent.

Statins seem to reduce AS in patients with dyslipidaemia [48,49,68,69,98], with or without CVD [99,100], with menopause [101], CKD [102], in overweight and obese [103], with T2DM [104], with T1DM [105], in healthy men [106], in patients with rheumatoid arthritis [107], and in elderly patients with arterial hypertension [108]. This effect of statins on AS is probably not a drug class effect, as suggested by the fact that the compound and the dose play a significant role in the degree of AS reduction [99-108]. It seems that potent statins, such as atorvastatin and rosuvastatin are more effective in reducing AS, reducing thus excessive CVD risk [109].

Polycystic ovary syndrome (PCOS) is the most common endocrine disorder affecting between $6 \%$ and $8 \%$ of women in reproductive age [110]. It is associated not only with reproductive and cosmetic problems, but also with significantly increased risk of metabolic dysfunction including IR with consequent compensatory hyperinsulinaemia, dyslipidaemia, systemic inflammation, increased oxidative stress, and endothelial dysfunction [110]. In the long-term, women with PCOS may develop T2DM, hypertension and atherosclerosis; ultimately, they are more likely to suffer from CVD and cerebrovascular diseases [110]. In patients with IR due to PCOS metformin improved aortic PWV and brachial PWV, CABP, endothelium-dependent and endothelium-independent vascular responses [111,112]. Metformin also reduced weight, waist circumference, and triglycerides and increased adiponectin [111]. Thus it seems that even short-term metformin therapy improves AS and endothelial function in young women with PCOS [111,112]. In addition, metformin has a considerable beneficial effect on endothelial function and AS in patients with MetS [113]. Furthermore, it has been demonstrated that increase in adiponectin level after treatment with the combination of two insulin sensitizers (pioglitazone plus metformin) may improve AS in patients with T2DM [114,115]. On the contrary the addition of liraglutide, a glucagon-like peptide-1 agonist (GLP-1 agonist), in patients with T2DM on metformin 
monotherapy, improved several CVD risk markers, beyond glycaemic control, but failed to improve AS [116]. Likewise, in a recent comparative study sitagliptin, but not glibenclamide, demonstrated a significant beneficial effect on BMI and triglycerides [117]. However, AS, BP, oxidative stress, and inflammatory status were not significantly changed by adding sitagliptin or glibenclamide to metformin-treated patients with T2DM [116]. From another ankle, metformin treatment was associated with significant decrease in AS after 1 year of treatment in patients with MetS and NAFLD, regardless of glycaemic control [118]. These beneficial effects suggest that metformin exhibit its vascular effects through glycaemic control-independent mechanisms [118]. In patients with T2DM three long-term different insulin regimens achieved overall good metabolic control with significant differences, however, in postprandial glucose increments [119]. The regimens achieving better vascular function (less AS) were those that induced a better postprandial glucose control [119]. Even after switching from premixed human insulin 30/70 containing rapid-acting insulin to biphasic insulin aspart 30/70 preparation [119], the later improved AS more than the former [120]. This improvement of AS may be associated with improvement of postprandial glycaemia [120].

\section{Limitations}

This review has the limitation of being narrative instead of systemic. However, conditions mentioned in introduction, mainly the lack of many original papers available that test the same hypothesis, did not allow us to write a systemic review and perform a meta-analysis.

\section{Conclusions}

AS and CABP are important independent CVD risk factors. A cluster of disease states related to MetS, they are either its components (arterial hypertension obesity, dyslipidaemia, dysglycaemia) or not (renal dysfunction, hyperuricaemia, hypercoaglutability, menopause, non alcoholic fatty liver disease, and obstructive sleep apnea) contribute, in an additive manner, to the increase of AS and thus to excessive CVD risk, often within a vicious cycle. Lifestyle measures, antihypertensive, hypolipidaemic, and hypoglycaemic drugs as well as CPAP in patients with OSA substantially contribute to the reduction of AS and finally to the decline in CVD risk.

There is a great unmet need for well planed, prospective, randomized, controlled, and long term interventional trials to assess the clinical benefit of regimens that improve AS. The examination of multifactorial interventions that showed reductions in CVD risk [121,122] might be even more useful and should also be tested.

AS and wave reflections have been widely investigated in old and/or hypertensive subjects for several reasons. In subjects $>50$ years of age, ventricular ejection fraction tends to be reduced, so that AS and amplitude and timing of wave reflections become the main determinants of increased systolic BP and PP. From the haemodynamic factors that influence PP, two have been shown to independently predict CVD risk: aortic stiffness, and early return of reflected waves to the heart, evaluated from pulse wave analysis. Finally, increased PP is a strong predictor of CVD risk in subjects with MetS, and/or all other cardio-metabolic risk factors analyzed above. It seems that in most cases of cardio-metabolic risk factors effective primary treatment of this factor reduces AS and PP. This is not always easy. Thus, symptomatic treatment of common determinants of AS, regardless of the initial cause, such as 
dyslipidaemia, $\mathrm{AH}$, diabetes, and smoking is the intervention of choice. Large long-term interventional studies are needed to investigate drug classes and particular drugs for their effect on AS and finally CVD and T2DM incidence in patients with a cluster of cardio-metabolic risk factors. Above all, we should abandon the dispute on the existence of MetS. The cluster of all these 12 cardio-metabolic risk factors analyzed above and their combinations indeed increases AS and CVD risk. This can not be disputed by anyone regardless of how we call it. A more rigorous review is needed to confirm the findings of this narrative review.

\section{Conflicts of Interest}

This review was written independently; no company or institution supported the authors financially or by providing a professional writer. Some of the authors have given talks, attended conferences and participated in trials and advisory boards sponsored by various pharmaceutical companies in the past, but they have no conflict of interest.

\section{References}

1. Laurent, S.; Alivon, M.; Beaussier, H.; Boutouyrie, P. Aortic stiffness as a tissue biomarker for predicting future cardiovascular events in asymptomatic hypertensive subjects. Ann. Med. 2012, 44 Suppl 1, S93-S97.

2. Palatini, P.; Casiglia, E.; Gąsowski, J.; Głuszek, J.; Jankowski, P.; Narkiewicz, K.; Saladini, F.; Stolarz-Skrzypek, K.; Tikhonoff, V.; Van Bortel, L.; et al. Arterial stiffness, central hemodynamics, and cardiovascular risk in hypertension. Vasc. Health. Risk. Manag. 2011, 7 , 725-739.

3. Meaume, S.; Benetos, A.; Henry, O.F.; Rudnichi, A.; Safar, M.E. Aortic pulse wave velocity predicts cardiovascular mortality in subjects $>70$ years of age. Arterioscler. Thromb. Vasc. Biol. 2001, 21, 2046-2050.

4. Boutouyrie, P.; Tropeano, A.I.; Asmar, R.; Gautier, I.; Benetos, A.; Lacolley, P.; Laurent, S. Aortic stiffness is an independent predictor of primary coronary events in hypertensive patients: a longitudinal study. Hypertension 2002, 39, 10-15.

5. Vlachopoulos, C.; Aznaouridis, K.; O'Rourke, M.F.; Safar, M.E.; Baou, K.; Stefanadis, C. Prediction of cardiovascular events and all-cause mortality with central haemodynamics: a systematic review and meta-analysis. Eur. Heart J. 2010, 31, 1865-1871.

6. Antonini-Canterin, F.; Carerj, S.; Di Bello, V.; Di Salvo, G.; La Carrubba, S.; Vriz, O.; Pavan, D.; Balbarini, A.; Nicolosi, G.L.; Research Group of the Italian Society of Cardiovascular Echography (SIEC). Arterial stiffness and ventricular stiffness: a couple of diseases or a coupling disease? A review from the cardiologist's point of view. Eur. J. Echocardiogr. 2009, 10, 36-43.

7. Vlachopoulos, C.; Alexopoulos, N.; Stefanadis, C. Aortic stiffness: Prime time for integration into clinical practice? Hellenic J. Cardiol. 2010, 51, 385-390.

8. Sassalos, K.; Vlachopoulos, C.; Alexopoulos, N.; Gialernios, T.; Aznaouridis, K.; Stefanadis, C. The acute and chronic effect of cigarette smoking on the elastic properties of the ascending aorta in healthy male subjects. Hellenic J. Cardiol. 2006, 47, 263-268. 
9. Cruickshank, K.; Riste, L.; Anderson, S.G.; Wright, J.S.; Dunn, G.; Gosling, R.G. Aortic pulsewave velocity and its relationship to mortality in diabetes and glucose intolerance: an integrated index of vascular function? Circulation 2002, 106, 2085-2090.

10. Vlachopoulos, C.; O'rourke, M. Genesis of the normal and abnormal arterial pulse. Curr. Probl. Cardiol. 2000, 25, 303-367.

11. Reference Values for Arterial Stiffness' Collaboration. Determinants of pulse wave velocity in healthy people and in the presence of cardiovascular risk factors: 'establishing normal and reference values'. Eur. Heart J. 2010, 31, 2338-2350.

12. Sugawara, J.; Hayashi, K.; Yokoi, T.; Cortez-Cooper, M.Y.; DeVan, A.E.; Anton, M.A.; Tanaka, H. Brachial-ankle pulse wave velocity: an index of central arterial stiffness? J. Hum. Hypertens. 2005, 19, 401-406.

13. Expert Panel on Detection, Evaluation, and Treatment of High Blood Cholesterol in Adults. Executive Summary of the Third Report of the National Cholesterol Education Program (NCEP) Expert Panel on Detection, Evaluation, and Treatment of High Blood Cholesterol in Adults (Adult Treatment Panel III). JAMA 2001, 285, 2486-2497.

14. Alberti, K.G.; Eckel, R.H.; Grundy, S.M.; Zimmet, P.Z.; Cleeman, J.I.; Donato, K.A.; Fruchart, J.C.; James, W.P.; Loria, C.M.; Smith, S.C.; et al. Harmonizing the metabolic syndrome: a joint interim statement of the International Diabetes Federation Task Force on Epidemiology and Prevention; National Heart, Lung, and Blood Institute; American Heart Association; World Heart Federation; International Atherosclerosis Society; and International Association for the Study of Obesity. Circulation 2009, 120, 1640-1645.

15. Lakka, H.M.; Laaksonen, D.E.; Lakka, T.A.; Niskanen, L.K.; Kumpusalo, E.; Tuomilehto, J.; Salonen, J.T. The metabolic syndrome and total and cardiovascular disease mortality in middle-aged men. JAMA 2002, 288, 2709-2716.

16. Ford, E.S., The metabolic syndrome and mortality from cardiovascular disease and all-causes: findings from the National Health and Nutrition Examination Survey II Mortality Study. Atherosclerosis 2004, 173, 309-314.

17. Kahn, R. Metabolic syndrome--what is the clinical usefulness? Lancet 2008, 371, 1892-1893.

18. Kahn, R.; Buse, J.; Ferrannini, E.; Stern, M.; American Diabetes Association; European Association for the Study of Diabetes. The metabolic syndrome: time for a critical appraisal: Joint statement from the American Diabetes Association and the European Association for the Study of Diabetes. Diabetes Care 2005, 28, 2289-2304.

19. IDF Consensus Worldwide Definition of the Metabolic Syndrome. Http://www.idf.org/webdata/docs/ IDF Meta def_final.Pdf (accessed on 18 November 2013).

20. Benetos, A.; Rudnichi, A.; Safar, M.; Guize, L. Pulse pressure and cardiovascular mortality in normotensive and hypertensive subjects. Hypertension 1998, 32, 560-564.

21. Nakanishi, N.; Suzuki, K.; Tatara, K. Clustered features of the metabolic syndrome and the risk for increased aortic pulse wave velocity in middle-aged Japanese men. Angiology 2003, 54, 551-559.

22. Brillante, D.G.; O'Sullivan, A.J.; Howes, L.G. Arterial stiffness in insulin resistance: the role of nitric oxide and angiotensin II receptors. Vasc. Health Risk. Manag. 2009, 5, 73-78. 
23. Sengstock, D.M.; Vaitkevicius, P.V.; Supiano, M.A. Arterial stiffness is related to insulin resistance in nondiabetic hypertensive older adults. J. Clin. Endocrinol. MeTable 2005, 90, $2823-2827$.

24. Seo, H.S.; Kang, T.S.; Park, S.; Park, H.Y.; Ko, Y.G.; Choi, D.; Jang, Y.; Chung, N. Insulin resistance is associated with arterial stiffness in nondiabetic hypertensives independent of metabolic status. Hypertens. Res. 2005, 28, 945-951.

25. Coutinho, M.; Gerstein, H.C.; Wang, Y.; Yusuf, S. The relationship between glucose and incident cardiovascular events. A metaregression analysis of published data from 20 studies of 95,783 individuals followed for 12.4 years. Diabetes Care 1999, 22, 233-240.

26. DECODE Study Group, t. E.D. E.G. Glucose tolerance and cardiovascular mortality: comparison of fasting and 2-hour diagnostic criteria. Arch. Intern. Med. 2001, 161, 397-405.

27. De Angelis, L.; Millasseau, S.C.; Smith, A.; Viberti, G.; Jones, R.H.; Ritter, J.M.; Chowienczyk, P.J. Sex differences in age-related stiffening of the aorta in subjects with type 2 diabetes. Hypertension 2004, 44, 67-71.

28. Ohnishi, H.; Saitoh, S.; Takagi, S.; Ohata, J.; Isobe, T.; Kikuchi, Y.; Takeuchi, H.; Shimamoto, K. Pulse wave velocity as an indicator of atherosclerosis in impaired fasting glucose: the Tanno and Sobetsu study. Diabetes Care 2003, 26, 437-440.

29. Ahluwalia, N.; Drouet, L.; Ruidavets, J.B.; Perret, B.; Amar, J.; Boccalon, H.; Hanaire-Broutin, H.; Ferrières, J. Metabolic syndrome is associated with markers of subclinical atherosclerosis in a French population-based sample. Atherosclerosis 2006, 186, 345-353.

30. Ferreira, I.; Boreham, C.A.; Twisk, J.W.; Gallagher, A.M.; Young, I.S.; Murray, L.J.; Stehouwer, C.D. Clustering of metabolic syndrome risk factors and arterial stiffness in young adults: the Northern Ireland Young Hearts Project. J. Hypertens. 2007, 25, 1009-1020.

31. Inoue, N.; Maeda, R.; Kawakami, H.; Shokawa, T.; Yamamoto, H.; Ito, C.; Sasaki, H. Aortic pulse wave velocity predicts cardiovascular mortality in middle-aged and elderly Japanese men. Circ. J. 2009, 73, 549-553.

32. Shin, J.Y.; Lee, H.R.; Lee, D.C. Increased arterial stiffness in healthy subjects with high-normal glucose levels and in subjects with pre-diabetes. Cardiovasc. Diabetol. 2011, 10, 30-34.

33. Webb, D.R.; Khunti, K.; Silverman, R.; Gray, L.J.; Srinivasan, B.; Lacy, P.S.; Williams, B.; Davies, M.J. Impact of metabolic indices on central artery stiffness: independent association of insulin resistance and glucose with aortic pulse wave velocity. Diabetologia 2010, 53, 11901198.

34. Park, J.S.; Nam, J.S.; Cho, M.H.; Yoo, J.S.; Ahn, C.W.; Jee, S.H.; Lee, H.S.; Cha, B.S.; Kim, K.R.; Lee, H.C. Insulin resistance independently influences arterial stiffness in normoglycemic normotensive postmenopausal women. Menopause 2010, 17, 779-784.

35. Simkova, A.; Bulas, J.; Balogova, S.; Reptova, A.; Kisa, B.; Luha, J.; Kinova, S. Metabolic syndrome and its effect on aortic stiffness in premenopausal women. Bratisl. Lek. Listy. 2013, $114,279-282$.

36. Thomas, G.; Sehgal, A.R.; Kashyap, S.R.; Srinivas, T.R.; Kirwan, J.P.; Navaneethan, S.D. Metabolic syndrome and kidney disease: a systematic review and meta-analysis. Clin. J. Am. Soc. Nephrol. 2011, 6, 2364-2373. 
37. Katsiki, N.; Athyros, V.G.; Karagiannis, A.; Mikhailidis, D.P. Characteristics Other Than the Diagnostic Criteria Associated with Metabolic Syndrome: an Overview. Curr. Vasc. Pharmacol. 2013, in press.

38. Wilson, A.C.; Mitsnefes, M.M. Cardiovascular disease in CKD in children: update on risk factors, risk assessment, and management. Am. J. Kidney Dis. 2009, 54, 345-360.

39. Shoji, T.; Abe, T.; Matsuo, H.; Egusa, G.; Yamasaki, Y.; Kashihara, N.; Shirai, K.; Kashiwagi, A. Committee of Renal and Peripheral Arteries, Japan Atherosclerosis. J. Atheroscler. Thromb. 2012, 19, 299-315.

40. Athyros, V.G.; Mikhailidis, D.P.; Liberopoulos, E.N.; Kakafika, A.I.; Karagiannis, A.; Papageorgiou, A.A.; Tziomalos, K.; Ganotakis, E.S.; Elisaf, M. Effect of statin treatment on renal function and serum uric acid levels and their relation to vascular events in patients with coronary heart disease and metabolic syndrome: a subgroup analysis of the GREek Atorvastatin and Coronary heart disease Evaluation (GREACE) Study. Nephrol. Dial. Transplant. 2007, 22, $118-127$.

41. Athyros, V.G.; Mikhailidis, D.P.; Papageorgiou, A.A.; Symeonidis, A.N.; Pehlivanidis, A.N.; Bouloukos, V.I.; Elisaf, M. The effect of statins versus untreated dyslipidaemia on renal function in patients with coronary heart disease. A subgroup analysis of the Greek atorvastatin and coronary heart disease evaluation (GREACE) study. J. Clin. Pathol. 2004, 57, 728-734.

42. Athyros, V.G.; Karagiannis, A.; Katsiki, N.; Mikhailidis, D.P. Statins in patients with renal dysfunction. Am. J. Cardiol. 2012, 109, 1537-1537.

43. Athyros, V.G.; Hatzitolios, A.I.; Karagiannis, A.; Savopoulos, C.; Katsiki, N.; Tziomalos, K.; Papagianni, A.; Kakafika, A.; Gossios, T.D.; Mikhailidis, D.P. Group, Improving the implementation of current guidelines for the management of major coronary heart disease risk factors by multifactorial intervention. the imperative renal analysis. Arch. Med. Sci. 2011, 7, 984-992.

44. Athyros, V.G.; Karagiannis, A.; Ganotakis, E.S.; Paletas, K.; Nicolaou, V.; Bacharoudis, G.; Tziomalos, K.; Alexandrides, T.; Liberopoulos, E.N.; Mikhailidis, D.P.; Assessing The Treatment Effect in Metabolic syndrome without Perceptible diabeTes (ATTEMPT) Collaborative Group. Association between the changes in renal function and serum uric acid levels during multifactorial intervention and clinical outcome in patients with metabolic syndrome. A post hoc analysis of the ATTEMPT study. Curr. Med. Res. Opin. 2011, 27, 1659-1668.

45. Safar, M.E.; London, G.M.; Plante, G.E. Arterial stiffness and kidney function. Hypertension 2004, 43, 163-168.

46. Katsiki, N.; Koumaras, C.; Athyros, V.G.; Karagiannis, A. Thinking beyond traditional cardiovascular risk factors: the role of arterial stiffness in targeting residual risk. Angiology 2012, 63, 9-11.

47. Koumaras, C.; Tzimou, M.; Stavrinou, E.; Griva, T.; Gossios, T.D.; Katsiki, N.; Athyros, V.G.; Mikhailidis, D.P.; Karagiannis, A. Role of antihypertensive drugs in arterial 'de-stiffening' and central pulsatile hemodynamics. Am. J. Cardiovasc. Drugs 2012, 12, 143-156. 
48. Kanaki, A.I.; Sarafidis, P.A.; Georgianos, P.I.; Kanavos, K.; Tziolas, I.M.; Zebekakis, P.E.; Lasaridis, A.N. Effects of low-dose atorvastatin on arterial stiffness and central aortic pressure augmentation in patients with hypertension and hypercholesterolemia. Am. J. Hypertens. 2013, 26, 608-616.

49. Sadat, U.; Howarth, S.P.; Usman, A.; Taviani, V.; Tang, T.Y.; Graves, M.J.; Gillard, J.H. Effect of low-and high-dose atorvastatin on carotid artery distensibility using carotid magnetic resonance imaging -a post-hoc sub group analysis of ATHEROMA (Atorvastatin Therapy: Effects On Reduction Of Macrophage Activity) Study. J. Atheroscler. Thromb. 2013, 20, 46-56.

50. Athyros, V.G.; Karagiannis, A.; Kakafika, A.; Elisaf, M.; Mikhailidis, D.P. Statins and renal function. Is the compound and dose making a difference? Nephrol. Dial. Transplant. 2007, 22, 963-964.

51. Katsiki, N.; Karagiannis, A.; Athyros, V.G.; Mikhailidis, D.P. Hyperuricaemia: more than just a cause of gout? J. Cardiovasc. Med. (Hagerstown) 2013, 14, 397-402.

52. Tsouli, S.G.; Liberopoulos, E.N.; Mikhailidis, D.P.; Athyros, V.G.; Elisaf, M.S. Elevated serum uric acid levels in metabolic syndrome: an active component or an innocent bystander? Metabolism 2006, 55, 1293-1301.

53. Bickel, C.; Rupprecht, H.J.; Blankenberg, S.; Rippin, G.; Hafner, G.; Daunhauer, A.; Hofmann, K.P.; Meyer, J. Serum uric acid as an independent predictor of mortality in patients with angiographically proven coronary artery disease. Am. J. Cardiol. 2002, 89, 12-17.

54. Hsu, P.F.; Chuang, S.Y.; Cheng, H.M.; Sung, S.H.; Ting, C.T.; Lakatta, E.G.; Yin, F.C.; Chou, P.; Chen, C.H. Associations of serum uric acid levels with arterial wave reflections and central systolic blood pressure. Int. J. Cardiol. 2013, 168, 2057-2063

55. Ishizaka, N.; Ishizaka, Y.; Toda, E.; Hashimoto, H.; Nagai, R.; Yamakado, M. Higher serum uric acid is associated with increased arterial stiffness in Japanese individuals. Atherosclerosis 2007, 192, 131-137.

56. Park, J.S.; Kang, S.; Ahn, C.W.; Cha, B.S.; Kim, K.R.; Lee, H.C. Relationships between serum uric acid, Adiponectin and arterial stiffness in postmenopausal women. Maturitas 2012, 73, 344-348.

57. Bian, S.; Guo, H.; Ye, P.; Luo, L.; Wu, H.; Xiao, W. Serum uric Acid level and diverse impacts on regional arterial stiffness and wave reflection. Iran. J. Public Health 2012, 41, 33-41.

58. Shin, J.Y.; Lee, H.R.; Shim, J.Y. Significance of high-normal serum uric acid level as a risk factor for arterial stiffness in healthy Korean men. Vasc. Med. 2012, 17, 37-43.

59. Khoshdel, A.R.; Carney, S.L.; Gillies, A. Circulatory syndrome: an evolution of the metabolic syndrome concept! Curr. Cardiol. Rev. 2012, 8, 68-76.

60. Devaraj, S.; Rosenson, R.S.; Jialal, I. Metabolic syndrome: an appraisal of the pro-inflammatory and procoagulant status. Endocrinol. Metab. Clin. North Am. 2004, 33, 431-453.

61. Nieuwdorp, M.; Stroes, E.S.; Meijers, J.C.; Büller, H. Hypercoagulability in the metabolic syndrome. Curr. Opin. Pharmacol. 2005, 5, 155-159.

62. Sagastagoitia, J.D.; Sáez, Y.; Vacas, M.; Narváez, I.; Sáez de Lafuente, J.P.; Molinero, E.; Magro, A.; Lafita, M.; Santos, M.; Escobar, A.; et al. Association between inflammation, lipid and hemostatic factors in patients with stable angina. Thromb. Res. 2007, 120, 53-59.

63. Angulo, P. Nonalcoholic fatty liver disease. N. Engl. J. Med. 2002, 346, 1221-1231. 
64. Lee, Y.J.; Shim, J.Y.; Moon, B.S.; Shin, Y.H.; Jung, D.H.; Lee, J.H.; Lee, H.R. The relationship between arterial stiffness and nonalcoholic fatty liver disease. Dig. Dis. Sci. 2012, 57, 196-203.

65. Salvi, P.; Ruffini, R.; Agnoletti, D.; Magnani, E.; Pagliarani, G.; Comandini, G.; Praticò, A.; Borghi, C.; Benetos, A.; Pazzi, P. Increased arterial stiffness in nonalcoholic fatty liver disease: the Cardio-GOOSE study. J. Hypertens. 2010, 28, 1699-1707.

66. Kim, B.J.; Kim, N.H.; Kim, B.S.; Kang, J.H. The association between nonalcoholic fatty liver disease, metabolic syndrome and arterial stiffness in nondiabetic, nonhypertensive individuals. Cardiology 2012, 123, 54-61.

67. Wilkinson, I.; Cockcroft, J.R. Cholesterol, Lipids and arterial stiffness. Adv. Cardiol. 2007, 44, 261-277.

68. Matsuo, T.; Iwade, K.; Hirata, N.; Yamashita, M.; Ikegami, H.; Tanaka, N.; Aosaki, M.; Kasanuki, H. Improvement of arterial stiffness by the antioxidant and anti-inflammatory effects of short-term statin therapy in patients with hypercholesterolemia. Heart Vessels 2005, 20, 8-12.

69. Wang, F.; Ye, P.; Luo, L.; Xiao, W.; Qi, L.; Bian, S.; Wu, H.; Sheng, L.; Xiao, T.; Xu, R. Association of serum lipids with arterial stiffness in a population-based study in Beijing. Eur. J. Clin. Invest. 2011, 41, 929-936.

70. van den Bogaard, B.; Holleboom, A.G.; Duivenvoorden, R.; Hutten, B.A.; Kastelein, J.J.; Hovingh, G.K.; Kuivenhoven, J.A.; Stroes, E.S.; van den Born, B.J. Patients with low HDLcholesterol caused by mutations in LCAT have increased arterial stiffness. Atherosclerosis 2012, $225,481-485$.

71. Urbina, E.M.; Khoury, P.R.; McCoy, C.E.; Dolan, L.M.; Daniels, S.R.; Kimball, T.R. Triglyceride to HDL-C ratio and increased arterial stiffness in children, adolescents, and young adults. Pediatrics 2013, 131, e1082-e1090.

72. Shimizu, Y.; Nakazato, M.; Sekita, T.; Kadota, K.; Yamasaki, H.; Takamura, N.; Aoyagi, K.; Maeda, T. Association of arterial stiffness and diabetes with triglycerides-to-HDL cholesterol ratio for Japanese men: The Nagasaki Islands Study. Atherosclerosis 2013, 228, 491-495.

73. Wilkinson, I.B.; Prasad, K.; Hall, I.R.; Thomas, A.; MacCallum, H.; Webb, D.J.; Frenneaux, M.P.; Cockcroft, J.R. Increased central pulse pressure and augmentation index in subjects with hypercholesterolemia. J. Am. Coll. Cardiol. 2002, 39, 1005-1011.

74. Litvin, A.; Sukmarova, Z.; Elfimova, E.; Aksenova, A.; Galitsin, P.; Rogoza, A.; Chazova, I. Effects of CPAP on "vascular" risk factors in patients with obstructive sleep apnea and arterial hypertension. Vasc. Health. Risk Manag. 2013, 9, 229-235.

75. Somers, V.K.; White, D.P.; Amin, R.; Abraham, W.T.; Costa, F.; Culebras, A.; Daniels, S.; Floras, J.S.; Hunt, C.E.; Olson, L.J.; et al. Sleep apnea and cardiovascular disease: an American Heart Association/American College of Cardiology Foundation Scientific Statement from the American Heart Association Council for High Blood Pressure Research Professional Education Committee, Council on Clinical Cardiology, Stroke Council, and Council on Cardiovascular Nursing. J. Am. Coll. Cardiol. 2008, 52, 686-717.

76. Jones, A.; Vennelle, M.; Connell, M.; McKillop, G.; Newby, D.E.; Douglas, N.J.; Riha, R.L. Arterial stiffness and endothelial function in obstructive sleep apnoea/hypopnoea syndrome. Sleep Med. 2013, 14, 428-432. 
77. Marin, J.M.; Carrizo, S.J.; Vicente, E.; Agusti, A.G. Long-term cardiovascular outcomes in men with obstructive sleep apnoea-hypopnoea with or without treatment with continuous positive airway pressure: an observational study. Lancet 2005, 365, 1046-1053.

78. Buchner, N.J.; Quack, I.; Stegbauer, J.; Woznowski, M.; Kaufmann, A.; Rump, L.C. Treatment of obstructive sleep apnea reduces arterial stiffness. Sleep Breath. 2012, 16, 123-133.

79. Vlachantoni, I.T.; Dikaiakou, E.; Antonopoulos, C.N.; Stefanadis, C.; Daskalopoulou, S.S.; Petridou, E.T. Effects of continuous positive airway pressure (CPAP) treatment for obstructive sleep apnea in arterial stiffness: a meta-analysis. Sleep Med. Rev. 2013, 17, 19-28.

80. Kato, M.; Kumagai, T.; Naito, R.; Maeno, K.; Kasagi, S.; Kawana, F.; Ishiwata, S.; Narui, K.; Kasai, T. Change in cardio-ankle vascular index by long-term continuous positive airway pressure therapy for obstructive sleep apnea. J. Cardiol. 2011, 58, 74-82.

81. Sugawara, J.; Otsuki, T.; Tanabe, T.; Hayashi, K.; Maeda, S.; Matsuda, M. Physical activity duration, intensity, and arterial stiffening in postmenopausal women. Am. J. Hypertens. 2006, 19, 1032-1036.

82. van de Laar, R.J.; Ferreira, I.; van Mechelen, W.; Prins, M.H.; Twisk, J.W.; Stehouwer, C.D. Lifetime vigorous but not light-to-moderate habitual physical activity impacts favorably on carotid stiffness in young adults: the Amsterdam growth and health longitudinal study. Hypertension 2010, 55, 33-39.

83. van de Laar, R.J.; Stehouwer, C.D.; van Bussel, B.C.; Prins, M.H.; Twisk, J.W.; Ferreira, I. Adherence to a Mediterranean dietary pattern in early life is associated with lower arterial stiffness in adulthood: the Amsterdam Growth and Health Longitudinal Study. J. Intern. Med. 2013, 273, 79-93.

84. van de Laar, R.J.; Stehouwer, C.D.; van Bussel, B.C.; te Velde, S.J.; Prins, M.H.; Twisk, J.W.; Ferreira, I. Lower lifetime dietary fiber intake is associated with carotid artery stiffness: the Amsterdam Growth and Health Longitudinal Study. Am. J. Clin. Nutr. 2012, 96, 14-23.

85. Lydakis, C.; Stefanaki, E.; Stefanaki, S.; Thalassinos, E.; Kavousanaki, M.; Lydaki, D. Correlation of blood pressure, obesity, and adherence to the Mediterranean diet with indices of arterial stiffness in children. Eur. J. Pediatr. 2012, 171, 1373-1382.

86. Tzima, N.; Pitsavos, C.; Panagiotakos, D.B.; Skoumas, J.; Zampelas, A.; Chrysohoou, C.; Stefanadis, C. Mediterranean diet and insulin sensitivity, lipid profile and blood pressure levels, in overweight and obese people; the Attica study. Lipids Health Dis. 2007, 6, 22-28.

87. Desroches, S.; Lapointe, A.; Ratté, S.; Gravel, K.; Légaré, F.; Thirsk, J. Interventions to enhance adherence to dietary advice for preventing and managing chronic diseases in adults: a study protocol. BMC Public Health 2011, 11, 111-114.

88. Dudenbostel, T.; Glasser, S.P. Effects of antihypertensive drugs on arterial stiffness. Cardiol. Rev. 2012, 20, 259-263.

89. Williams, B.; Lacy, P.S.; Thom, S.M.; Cruickshank, K.; Stanton, A.; Collier, D.; Hughes, A.D.; Thurston, H.; O'Rourke, M.; Investigators, C.; et al. Differential impact of blood pressurelowering drugs on central aortic pressure and clinical outcomes: principal results of the Conduit Artery Function Evaluation (CAFE) study. Circulation 2006, 113, 1213-1225.

90. Duprez, D.A. Is vascular stiffness a target for therapy? Cardiovasc. Drugs. Ther. 2010, 24, 305-310. 
91. Najjar, S.S.; Scuteri, A.; Shetty, V.; Wright, J.G.; Muller, D.C.; Fleg, J.L.; Spurgeon, H.P.; Ferrucci, L.; Lakatta, E.G. Pulse wave velocity is an independent predictor of the longitudinal increase in systolic blood pressure and of incident hypertension in the Baltimore Longitudinal Study of Aging. J. Am. Coll. Cardiol. 2008, 51, 1377-1383.

92. Jin, Y.; Thijs, L.; Richart, T.; Li, Y.; Dolan, E.; Wang, J.G.; Protogerou, A.; O'Brien, E.; Staessen, J.A.; Safar, M.E. Responses of the ambulatory arterial stiffness index and other measures of arterial function to antihypertensive drugs. Hypertens. Res. 2011, 34, 489-495.

93. Ng, K.; Butlin, M.; Avolio, A.P. Persistent effect of early, brief angiotensin-converting enzyme inhibition on segmental pressure dependency of aortic stiffness in spontaneously hypertensive rats. J. Hypertens. 2012, 30, 1782-1790.

94. Ong, K.T.; Delerme, S.; Pannier, B.; Safar, M.E.; Benetos, A.; Laurent, S.; Boutouyrie, P, Aortic stiffness is reduced beyond blood pressure lowering by short-term and long-term antihypertensive treatment: a meta-analysis of individual data in 294 patients. J. Hypertens. 2011, 29, 1034-1042.

95. Pende, A.; Dallegri, F. Renin-angiotensin antagonists: therapeutic effects beyond blood pressure control? Curr. Pharm. Des. 2012, 18, 1011-1020.

96. Shahin, Y.; Khan, J.A.; Chetter, I. Angiotensin converting enzyme inhibitors effect on arterial stiffness and wave reflections: a meta-analysis and meta-regression of randomised controlled trials. Atherosclerosis 2012, 221, 18-33.

97. Williams, B.; Lacy, P.S.; Cruickshank, J.K.; Collier, D.; Hughes, A.D.; Stanton, A.; Thom, S.; Thurston, H.; CAFE and ASCOT Investigators. Impact of statin therapy on central aortic pressures and hemodynamics: principal results of the Conduit Artery Function Evaluation-LipidLowering Arm (CAFE-LLA) Study. Circulation 2009, 119, 53-61.

98. Hongo, M.; Kumazaki, S.; Izawa, A.; Hidaka, H.; Tomita, T.; Yazaki, Y.; Kinoshita, O.; Ikeda, U. Low-dose rosuvastatin improves arterial stiffness in high-risk Japanese patients with dyslipdemia in a primary prevention group. Circ. J. 2011, 75, 2660-2667.

99. Hongo, M.; Tsutsui, H.; Mawatari, E.; Hidaka, H.; Kumazaki, S.; Yazaki, Y.; Takahashi, M.; Kinoshita, O.; Ikeda, U. Fluvastatin improves arterial stiffness in patients with coronary artery disease and hyperlipidemia: a 5-year follow-up study. Circ. J. 2008, 72, 722-728.

100. Kontopoulos, A.G.; Athyros, V.G.; Pehlivanidis, A.N.; Demitriadis, D.S.; Papageorgiou, A.A.; Boudoulas, $H$. Long-term treatment effect of atorvastatin on aortic stiffness in hypercholesterolaemic patients. Curr. Med. Res. Opin. 2003, 19, 22-27.

101. Igase, M.; Kohara, K.; Tabara, Y.; Nagai, T.; Ochi, N.; Kido, T.; Ochi, M.; Miki, T. Low-dose rosuvastatin improves the functional and morphological markers of atherosclerosis in asymptomatic postmenopausal women with dyslipidemia. Menopause 2012, 19, 1294-1299.

102. Fassett, R.G.; Robertson, I.K.; Ball, M.J.; Geraghty, D.P.; Sharman, J.E.; Coombes, J.S. Effects of atorvastatin on arterial stiffness in chronic kidney disease: a randomised controlled trial. $J$. Atheroscler. Thromb. 2010, 17, 235-241.

103. Orr, J.S.; Dengo, A.L.; Rivero, J.M.; Davy, K.P. Arterial destiffening with atorvastatin in overweight and obese middle-aged and older adults. Hypertension 2009, 54, 763-768. 
104. Mukherjee, S.; Mukhopadhyay, P.; Pandit, K.; Chowdhury, S. Atorvastatin improves arterial stiffness in normotensive normolipidaemic persons with type 2 diabetes. J. Indian. Med. Assoc. 2008, 106, 716-719.

105. Haller, M.J.; Stein, J.M.; Shuster, J.J.; Theriaque, D.; Samyn, M.M.; Pepine, C.; Silverstein, J.H. Pediatric Atorvastatin in Diabetes Trial (PADIT): a pilot study to determine the effect of atorvastatin on arterial stiffness and endothelial function in children with type 1 diabetes mellitus. J. Pediatr. Endocrinol. MeTable 2009, 22, 65-68.

106. Lunder, M.; Janić, M.; Habjan, S.; Sabovič, M. Subtherapeutic, low-dose fluvastatin improves functional and morphological arterial wall properties in apparently healthy, middle-aged males--a pilot study. Atherosclerosis 2011, 215, 446-451.

107. Tam, L.S.; Li, E.K.; Shang, Q.; Tomlinson, B.; Lee, V.W.; Lee, K.K.; Li, M.; Kuan, W.P.; Li, T.K.; Tseung, L.; et al. Effects of rosuvastatin on subclinical atherosclerosis and arterial stiffness in rheumatoid arthritis: a randomized controlled pilot trial. Scand. J. Rheumatol. 2011, 40, 411-421.

108. Wang, J.; Xu, J.; Zhou, C.; Zhang, Y.; Xu, D.; Guo, Y.; Yang, Z. Improvement of arterial stiffness by reducing oxidative stress damage in elderly hypertensive patients after 6 months of atorvastatin therapy. J. Clin. Hypertens. (Greenwich) 2012, 14, 245-249.

109. Toyama, K.; Sugiyama, S.; Oka, H.; Iwasaki, Y.; Sumida, H.; Tanaka, T.; Tayama, S.; Jinnouchi, H.; Ogawa, H. Combination treatment of rosuvastatin or atorvastatin, with regular exercise improves arterial wall stiffness in patients with coronary artery disease. PLoS One 2012, 7, e41369-e41376.

110. Duleba, A.J. Medical management of metabolic dysfunction in PCOS. Steroids 2012, 77, 306-311.

111. Agarwal, N.; Rice, S.P.; Bolusani, H.; Luzio, S.D.; Dunseath, G.; Ludgate, M.; Rees, D.A. Metformin reduces arterial stiffness and improves endothelial function in young women with polycystic ovary syndrome: a randomized, Placebo-controlled, Crossover trial. J. Clin. Endocrinol. MeTable 2010, 95, 722-730.

112. Kaya, M.G.; Calapkorur, B.; Karaca, Z.; Yildirim, S.; Celik, A.; Akpek, M.; Unluhizarci, K.; Kelestimur, F. The effects of treatment with drospirenone/ethinyl oestradiol alone or in combination with metformin on elastic properties of aorta in women with polycystic ovary syndrome. Clin. Endocrinol. (Oxf) 2012, 77, 885-892.

113. Meaney, E.; Vela, A.; Samaniego, V.; Meaney, A.; Asbún, J.; Zempoalteca, J.C.; Elisa, Z.N.; Emma, M.N.; Guzman, M.; Hicks, J.; et al. Metformin, arterial function, intima-media thickness and nitroxidation in metabolic syndrome: the mefisto study. Clin. Exp. Pharmacol. Physiol. 2008, 35, 895-903.

114. Araki, T.; Emoto, M.; Teramura, M.; Yokoyama, H.; Mori, K.; Hatsuda, S.; Maeno, T.; Shinohara, K.; Koyama, H.; Shoji, T.; Inaba, M.; Nishizawa, Y. Effect of adiponectin on carotid arterial stiffness in type 2 diabetic patients treated with pioglitazone and metformin. Metabolism 2006, 55, 996-1001.

115. Kiyici, S.; Ersoy, C.; Kaderli, A.; Fazlioglu, M.; Budak, F.; Duran, C.; Gul, O.O.; Sigirli, D.; Baran, I.; Tuncel, E.; et al. Effect of rosiglitazone, metformin and medical nutrition treatment on arterial stiffness, serum MMP-9 and MCP-1 levels in drug naive type 2 diabetic patients. Diabetes Res. Clin. Pract. 2009, 86, 44-50. 
116. Forst, T.; Michelson, G.; Ratter, F.; Weber, M.M.; Anders, S.; Mitry, M.; Wilhelm, B.; Pfützner, A. Addition of liraglutide in patients with Type 2 diabetes well controlled on metformin monotherapy improves several markers of vascular function. Diabet. Med. 2012, 29, 1115-1118.

117. Koren, S.; Shemesh-Bar, L.; Tirosh, A.; Peleg, R.K.; Berman, S.; Hamad, R.A.; Vinker, S.; Golik, A.; Efrati, S. The effect of sitagliptin versus glibenclamide on arterial stiffness, blood pressure, lipids, and inflammation in type 2 diabetes mellitus patients. Diabetes Technol. Ther. 2012, 14, 561-567.

118. Shargorodsky, M.; Omelchenko, E.; Matas, Z.; Boaz, M.; Gavish, D. Relation between augmentation index and adiponectin during one-year metformin treatment for nonalcoholic steatohepatosis: effects beyond glucose lowering? Cardiovasc. Diabetol. 2012, 11, 61-68.

119. Bibra, H.; Siegmund, T.; Ceriello, A.; Volozhyna, M.; Schumm-Draeger, P.M. Optimized postprandial glucose control is associated with improved cardiac/vascular function-comparison of three insulin regimens in well-controlled type 2 diabetes. Horm. Metab. Res. 2009, 41, 109-115.

120. Ohira, M.; Endo, K.; Oyama, T.; Yamaguchi, T.; Ban, N.; Kawana, H.; Nagayama, D.; Nagumo, A.; Saiki, A.; Murano, T.; et al. Improvement of postprandial hyperglycemia and arterial stiffness upon switching from premixed human insulin 30/70 to biphasic insulin aspart 30/70. Metabolism 2011, 60, 78-85.

121. Athyros, V.G.; Mikhailidis, D.P.; Papageorgiou, A.A.; Bouloukos, V.I.; Pehlivanidis, A.N.; Symeonidis, A.N.; Elisaf, M.; Group, G.S.C. Effect of statins and ACE inhibitors alone and in combination on clinical outcome in patients with coronary heart disease. J. Hum. Hypertens. 2004, 18, 781-788.

122. Athyros, V.G.; Mikhailidis, D.P.; Papageorgiou, A.A.; Bouloukos, V.I.; Pehlivanidis, A.N.; Symeonidis, A.N.; Kakafika, A.I.; Daskalopoulou, S.S.; Elisaf, M. Effect of statins and aspirin alone and in combination on clinical outcome in dyslipidaemic patients with coronary heart disease. A subgroup analysis of the GREACE study. Platelets 2005, 16, 65-71.

(C) 2013 by the authors; licensee MDPI, Basel, Switzerland. This article is an open access article distributed under the terms and conditions of the Creative Commons Attribution license (http://creativecommons.org/licenses/by/3.0/). 